\title{
Demokratischer Smog? Eine empirische Untersuchung zum Zusammenhang zwischen Sozialschicht und Umweltbelastungen
}

\author{
Andreas Diekmann · Reto Meyer
}

Zusammenfassung: Seit langem gibt es in den USA eine Debatte über „Umweltgerechtigkeit““ In den letzten Jahren wurde der Frage nach dem ,Sozialgradienten“ der Umweltbelastung auch im deutschsprachigen Raum nachgegangen. In „Umweltsurveys“ wurden allerdings nur subjektive Angaben zur Umweltbelastung herangezogen. Wurden objektive Maße verwendet, so sind die Studien nur auf kleinere geographische Gebiete beschränkt. Demgegenüber verwenden wir „objektive“ Daten zur Umweltverschmutzung (Stickstoffdioxid, Feinstaub, Ozon, Lärmbelastung), die mit den „subjektiven“ Angaben befragter Haushalte des „Schweizer Umweltsurveys 2007“ $(\mathrm{N}=3369)$ in einem Geoinformationssystem (GIS) verknüpft wurden. Mit der Kombination von Befragtendaten und GIS-kodierten Daten eröffnet sich nicht nur für die empirische Umweltsoziologie eine neue und interessante Perspektive: Die Berücksichtigung des räumlichen Kontextes bei der statistischen Analyse von Umfragedaten. Mit bivariaten und multivariaten Methoden werden die Zusammenhänge zwischen der objektiven Umweltbelastung am Wohnort und dem Sozialstatus, der Nationalität und der subjektiven Wahrnehmung von Umweltbelastungen untersucht. Subjektive und objektive Umweltbelastungen sind erwartungsgemäß positiv korreliert mit der

(C) VS Verlag für Sozialwissenschaften 2010

Gefördert wurde der Schweizer Umweltsurvey 2007 durch den Schweizerischen Nationalfonds (Projekt: 100012-107835). Das Projekt wurde vom Bundesamt für Umwelt (BAFU), den Kantonen Basel-Stadt (Amt für Umwelt und Energie) und Zürich (Amt für Abfall, Wasser, Energie und Luft), den Zentralschweizer Kantonen (Umweltämter) und dem Umwelt- und Gesundheitsschutz der Stadt Zürich unterstützt. Ferner wurde das Projekt finanziell und bezüglich der Stichprobenauswahl vom Bundesamt für Statistik (BFS) gefördert. Das BFS hat keinen Einfluss auf die verwendete Methode und die daraus resultierenden Ergebnisse. Wir danken den Abteilungen für Luftreinhaltung und Lärmbekämpfung des BAFUs für die Daten zu den objektiven Umweltbelastungen. Ebenfalls gilt unser Dank den beiden anonymen Gutachtern und Herrn Thomas Künzle von Meteotest für ihre wertvollen Hinweise. Thomas Künzle danken wir für die Beschreibung der Modelle zur Berechnung der Schadstoffbelastung im Anhang 1.

A. Diekmann $(\bowtie) \cdot$ R. Meyer

Soziologie, ETH Zürich, Clausiusstraße 50,

8092 Zürich, Schweiz

E-Mail: diekmann@soz.gess.ethz.ch

R. Meyer

E-Mail: reto.meyer@soz.gess.ethz.ch 
interessanten Ausnahme von Ozon. Überraschend ist, dass die Indikatoren des Sozialstatus nicht signifikant (Einkommen) oder sogar signifikant positiv (Bildung) mit der objektiven Umweltbelastung korrelieren. In der multivariaten Analyse zeigt sich ein signifikant negativer Zusammenhang mit dem Einkommen, der aber relativ schwach ausgeprägt ist. Ausländer aus nicht-westlichen Ländern haben eine höhere Umweltbelastung als Inländer, wobei diese Differenz aber geringer ist als der Unterschied in der Umweltbelastung von urbanen Gebieten gegenüber Haushalten auf dem Land. Andere Länder als die Schweiz werden vermutlich stärkere Unterschiede aufweisen, insbesondere wenn die Segregation von inländischer und ausländischer Bevölkerung stark ausgeprägt ist.

Schlüsselwörter: Umweltgerechtigkeit · Soziale Verteilung der Umweltbelastung · Umweltsurvey · Survey- und GIS-Daten

\title{
Democratic smog? An empirical study on the correlation between social class and environmental pollution
}

\begin{abstract}
For years the public and scientific debate about Environmental Justice was mostly confined to the U.S. Only recently, the question about the existence and strength of the "social gradient" of environmental pollution has entered the European debate. Earlier research simply records subjective perceptions of pollution and correlates them with indicators of social status. Objective measures of environmental quality are seldom used, and even then only in studies of small geographic areas. In contrast, the present study uses various objective measures of air pollution (nitrogen dioxide, particulate matter, ozone) and road traffic noise (day, night) and assigns them to the respondents of the Swiss Environmental Survey $2007(\mathrm{~N}=3$ 369) using a geographic information system (GIS). The combination of objective GIS coded data with subjective measures allows for a new approach in Environmental Sociology that takes spatial context into account, which is often neglected in sociological studies. Using bivariate and multivariate statistical methods this objective data on pollution is related to indicators of social stratification such as income, education and nationality as well as the subjective perception of pollution. Subjective and objective measures of pollution are positively correlated with the interesting exception of ozone. Surprisingly, and contrary to the expectations, income is not significantly correlated with the pollutants considered and there is even a significant and positive correlation with education. In the multivariate analysis, however, a significant and negative correlation with income is observed, although this effect is fairly weak. Also, foreigners from Non-Western countries suffer from a higher burden of environmental pollution. However, in comparison to social and minority status differences in environmental impact between urban areas and the countryside are much more pronounced. We suppose that problems of environmental justice may be more accentuated in countries with a higher degree of segregation than in the Swiss population.
\end{abstract}

Keywords: Environmental justice - Social status and environmental impact · Environmental survey · GIS · Data linkage

\section{Einleitung: Umweltgerechtigkeit und der „Sozialgradient“ von Umweltbelastungen}

„Environmental Justice“ lesen wir auf dem Banner von Protestbewegungen und Bürgerinitiativen in den USA, die unter dieser Parole Umweltbelastungen und Gesundheitsrisiken anprangern, denen in besonderem Maße benachteiligte Sozialschichten und ethnische 
Minoritäten ausgesetzt sind. Lokale Industrieemissionen, Lärm, Abfälle, Deponien mit toxischem Material treten gehäuft dort auf, wo der politische Widerstand gering, dagegen Armut, Arbeitslosigkeit und der Anteil ethnischer Minoritäten hohe Ausprägungen aufweisen, lautet die Klage. Die Proteste und Initiativen fanden auch in der wissenschaftlichen Forschung Resonanz. Empirische Untersuchungen zur „Umweltgerechtigkeit“ liefern mit Fallstudien und statistischen Daten Belege für die kumulativen Belastungen unterer Sozialschichten in den hoch segregierten urbanen Siedlungen Nordamerikas (Maschewsky 2001; Evans u. Kantrowitz 2002; O’Neill et al. 2003; Ash u. Fetter 2004; Maschewsky 2004). Allerdings gibt es bei einzelnen Indikatoren auch negative Ergebnisse. So findet Atlas (2002) für die USA keinen Beleg dafür, dass im Umkreis toxischer Deponien überwiegend ärmere Bevölkerungsgruppen und Angehörige von Minoritäten siedeln. Unstrittig ist die Abhängigkeit der Umweltqualität vom Einkommen im globalen Maßstab. Gravierende Luftverschmutzung, Knappheit und Zugang zu Trinkwasser, mangelnde Wasserqualität und Hygiene sind Sorgen, die insbesondere arme Länder plagen. Bei einigen Umweltindikatoren findet man auch einen umgekehrt u-förmigen Zusammenhang zwischen der lokalen Umweltqualität und dem Pro-Kopfeinkommen, die so genannte „,environmental Kuznets-curve“ (Grossman u. Krüger 1995; Ehrhardt-Martinez et al. 2002; kritisch York et al. 2003). Die Umweltbelastungen steigen demnach mit dem Aufstieg zu Schwellenländern und sinken wieder, wenn Staaten mehr Wohlstand erwerben, um die Folgen ungezügelter Industrialisierung abzufedern.

Urbane Segregation und die damit einhergehenden unterschiedlichen Umweltbelastungen nach Sozialschicht und Nationalität sind auch ein Thema für europäische Gesellschaften. Jede Stadt hat ihr ,,besseres“, grünes und ruhiges Viertel, in Deutschland wegen der meteorologischen Verhältnisse oft im Westen der Städte. Sehr wahrscheinlich hat sich die Stärke und Varianz der Belastungen mit dem Wandel von der Industrie- zur Dienstleistungsgesellschaft verändert, wobei aber auch neue Umweltprobleme wie wachsender Verkehrslärm, Fluglärm und verkehrsbedingte Feinstaubemissionen hinzugekommen sind. $\mathrm{Ob}$ und in welchem Ausmaß sich der „Sozialgradient“ von Umweltbelastungen verringert oder verschärft hat und wie stark der Zusammenhang heute überhaupt ausgeprägt ist, sind brisante und für die Umwelt- und Gesundheitspolitik wichtige Fragen. Dagegen gibt es in Deutschland, Österreich und der Schweiz nur vereinzelte Untersuchungen, die meist nur im lokalen Rahmen soziale Lagen und „objektive“ Messdaten der Umweltqualität miteinander in Beziehung setzen (Überblick in Mielck u. Heinrich 2002; Bolte u. Mielck 2004; Bolte u. Kohlhuber 2008, Hornberg u. Pauli 2009, vgl. auch Elvers 2007). Häufig werden auch nur subjektive Wahrnehmungen, wie sie in „Umweltsurveys“ erfasst werden, mit Indikatoren der sozialen Lage korreliert (Grunenberg u. Kuckartz 2003). Ausschließlich subjektive Befragungsdaten zur Wahrnehmung der Umweltqualität mögen für manche Fragestellungen von Interesse sein, über den Sozialgradienten von Umweltbelastungen geben sie allerdings kein zutreffendes Bild. So findet man positive Zusammenhänge mit der ,grünen“ Orientierung oder dem Bildungsgrad, aber nicht unbedingt, weil die Umweltbelastung höher ist, sondern weil Umweltprobleme sensibler wahrgenommen werden (Preisendörfer 1999, S. 178 ff.).

Welche Gründe können dafür geltend gemacht werden, dass es zu einer erhöhten Konzentration von Umweltbelastungen in der Umgebung der Wohnorte unterer Sozialschichten kommt? Zwei Mechanismen können angeführt werden. Erstens könnte es sein, 
dass Verkehrsprojekte, Industrieansiedlungen und die Deponierung von Schadstoffen von kommunalen oder anderen Entscheidungsträgern eher in den ärmeren Wohngebieten geplant werden, weil dort geringerer politischer Widerstand zu erwarten ist. Hinzu kommt noch, dass Entscheidungsträger bemüht sein könnten, ihre eigenen, im Durchschnitt wohlhabenderen Wohngebiete zu verschonen. Zweitens ist anzunehmen, dass die Umweltqualität eines Wohnorts einen Einfluss auf die Mietpreise und die Preise von Wohnungseigentum ausübt. Via Marktmechanismus sollte das Ausmaß von Umweltlasten durch die Preise reflektiert werden. $\mathrm{Ob}$ in Zürich oder München, in Flughafennähe sind die Mieten und Bodenpreise in der Regel geringer als am Starnberger See oder der „Goldküste“ des Zürichsees. Aus beiden Mechanismen folgt der inverse Zusammenhang zwischen sozialer Schichtung und Umweltbelastungen.

In der Soziologie, die von ihrer Profession her Problemen sozialer Ungleichheit sonst große Aufmerksamkeit widmet, gab es zumindest in Deutschland kaum Anstöße zu empirischen Studien in der Tradition der Forschung über Umweltgerechtigkeit. Mit der These der Enthierarchisierung von Modernisierungsrisiken wurde in der Soziologie sogar, allerdings ohne systematische empirische Belege, die Gegenthese zur Tradition der Forschung über Umweltgerechtigkeit postuliert. Ulrich Beck (1986, S. 48) hat dies in der „Risikogesellschaft“ auf den Punkt gebracht: „Not ist hierarchisch, Smog ist demokratisch“. In der Sozialmedizin wurde dagegen zu diesem Thema weit mehr empirisch geforscht als in der Soziologie. Oft sind es Studien über den Gesundheitszustand von Kindern, in denen die Wohnsituation und soziale Merkmale der Eltern miterfasst wurden. So haben Sozialmediziner in Schuleingangsuntersuchungen festgestellt, dass die soziale Lage, die Wohnverhältnisse und umweltbedingte Gesundheitsrisiken korreliert sind (Mielck u. Heinrich 2002). In der Schweiz ist Braun-Fahrländer (2004) der Frage nach dem Zusammenhang zwischen Sozialschicht und Umweltbelastung bei Basler Kindern nachgegangen. Der Befund lautet, dass Kinder aus unteren Schichten stärker von Verkehrslärm und Gesundheitsrisiken durch Schadstoffe in der Innenluft (u. a. bedingt durch das Rauchen der Eltern) betroffen sind als Kinder aus oberen Sozialschichten. Es gibt punktuelle Untersuchungen, aber keine systematische, landesweite Erhebung, die Indikatoren der Sozialschicht mit Indikatoren der Umweltbelastung am Wohnort verknüpft.

Unser Anliegen ist es, erstmals Ergebnisse aus einer landesweiten Untersuchung in der Schweiz zu berichten und zur Diskussion zu stellen. Auch methodisch ist unsere Studie von Interesse. Wir verknüpfen zur Beantwortung der Fragestellung nach der Verteilung von Umweltlasten die Befragungsdaten des Schweizer Umweltsurveys mit den Daten der Luft- und Lärmbelastung in einem Geo-Informationssystem (GIS). Der Schweizer Umweltsurvey ist eine landesweite Erhebung zu einem breiten Spektrum umweltbezogener Themen und sozialdemografischer Merkmale. Kataster verschiedener Indikatoren der Luftverschmutzung und der Lärmbelastung liegen in elektronischer Form bei eidgenössischen Bundesämtern vor. Werden den Adressen der Befragten Landeskoordinaten zugeschrieben, können beide Datensätze, die Individualdaten des Umweltsurveys und die raumbezogenen Daten zur Umweltbelastung, in einem Geo-Informationssystem verknüpft und auf der Mikroebene der Befragten analysiert werden. Die Soziologie vernachlässigt in empirischen Analysen oft die räumliche Dimension. Mit der Geo-Referenzierung von Mikrodaten aus Befragungen und ihrer Verknüpfung mit anderen Datenquellen in einem 
Geo-Informationssystem bietet sich die Gelegenheit, den räumlichen und sozialen Kontext von Haushalten bei soziologischen Untersuchungen zu berücksichtigen.

In Abschn. 2 erläutern wir die Daten und die Methode der Untersuchung. In Abschn. 3 werden die Ergebnisse bivariater Korrelationen und multivariater Analysen mit verschiedenen Indikatoren der Umweltbelastungen berichtet. Unabhängige Variablen sind Indikatoren der sozialen Schichtzugehörigkeit, abhängige Variablen sind Indikatoren der Umweltbelastung. Neben den Analysen mit den physikalischen Messungen von Luftschadstoffen und Lärm interessiert in Abschn. 4 auch die subjektive Wahrnehmung der Umweltqualität. In Abschn. 5 werden die Befunde diskutiert und weitere Forschungsfragen angesprochen.

\section{Daten und Methode}

Der Schweizer Umweltsurvey basiert auf einer zweistufigen Zufallsstichprobe aus der erwachsenen Wohnbevölkerung der Schweiz mit registrierten Telefonanschlüssen. Die ausgewählten Haushalte wurden zuvor angeschrieben und um die Mitarbeit an der Studie gebeten. Die Studie wurde als Untersuchung über „Lebensverhältnisse in der Schweiz“ und nicht als „Umweltsurvey“ angekündigt, um auszuschließen, dass vorwiegend überdurchschnittlich an Umweltfragen Interessierte für die Mitarbeit motiviert wurden.

Im Zeitraum von November 2006 bis März 2007 wurden telefonische Interviews mit 3369 zufällig ausgewählten Haushalten geführt. Die zu befragende Person wurde wiederum zufällig aus den über 18 Jahre alten Mitgliedern des Haushalts ausgewählt und in deutscher, französischer oder italienischer Sprache interviewt. Ausländer, die zur Wohnbevölkerung zählen, wurden berücksichtigt, sofern sie in einer der drei Sprachen Auskunft geben konnten. Die Ausschöpfungsquote beträgt 52\% (strikte Berechnung gemäß dem Standard der American Association für Public Opinion Research). An das telefonische Interview mit einer durchschnittlichen Dauer von 37 Minuten schloss sich eine schriftliche Nachbefragung an, wobei mit dem Fragebogen ein kleines Geschenk verschickt wurde. $83 \%$ der telefonisch interviewten Personen haben sich an der schriftlichpostalischen Umfrage beteiligt (Diekmann u. Meyer 2008).

Bei der zweistufigen Zufallsauswahl haben Personen in größeren Haushalten eine geringere Chance, in die Stichprobe zu gelangen. Außerdem wurde die Stichprobe regional geschichtet. Bei Auswertungen auf der Befragtenebene können wir für die unterschiedlichen Inklusionswahrscheinlichkeiten mit einem Designgewicht korrigieren. Auf der Haushaltsebene korrigiert das Designgewicht nur für die regionale Schichtung. Nur bei den deskriptiven Daten verwenden wir Designgewichte. Bei den Schätzungen mittels multivariater Regression verwenden wir die ungewichteten Daten. Um sicher zu gehen, prüfen wir aber durch Vergleichsrechnungen mit Gewichtung die Robustheit der Ergebnisse.

Als Indikatoren der Sozialschicht werden das Haushaltseinkommen und der Bildungsgrad herangezogen. Hinzu kommt das Merkmal Nationalität, wobei wir vier Kategorien berücksichtigen: 1. Befragte mit Schweizer Staatsbürgerschaft, 2. Westeuropa/Nordamerika, 3. Südeuropa (Griechenland, Italien, Portugal, Spanien), 4. Andere Staaten (Balkan, Osteuropa, Asien, Südamerika, Afrika). Aus dem Haushaltseinkommen wird durch 
geeignete Gewichtung mit der Haushaltsgröße das Äquivalenzeinkommen konstruiert, das wir in den statistischen Analysen verwenden. Berücksichtigt wurde ferner der höchste Bildungsabschluss der befragten Person und, sofern im gleichen Haushalt lebend, der Bildungsabschluss des Partners oder der Partnerin. Der höchste Bildungsabschluss wurde sodann in Bildungsjahre (nach Auskunft des Bundesamts für Statistik) ${ }^{1}$ umgewandelt. Wir erhalten dann zwei Bildungsvariablen: Die Bildungsjahre des Befragten und den „Bildungsstatus des Haushalts“. In Partnerhaushalten entspricht dieser den Bildungsjahren des Partners mit dem höheren Abschluss. Weitere soziodemografische Merkmale sind Alter, Geschlecht, Gemeindegröße, Sprachregion, die Haushaltsgröße und das Merkmal, ob Kinder im Haushalt leben. Bei Mietern wird die Höhe der Miete einen Einfluss auf die Wohnortwahl haben. Wohnungseigentümer werden bei der Anschaffung besonderes Augenmerk auf den Standort richten und größere Umweltbelastungen eher meiden. Beide Variablen, Miethöhe und Wohnungseigentum, wurden im Umweltsurvey erhoben. Weiterhin wurde nach der subjektiven Wahrnehmung der Umweltqualität gefragt. Die subjektive Bewertung von Lärm und Luftverschmutzung wurde mit fünfstufigen Skalen im Telefoninterview und mit zehnstufigen Skalen in der schriftlichen Befragung erfasst. Über die Operationalisierung der Variablen des Umweltsurveys und die deskriptive Statistik informiert Tab. 1.

Indikatoren der Luftverschmutzung sind Stickstoffdioxid $\left(\mathrm{NO}_{2}\right)$, Feinstaub unterschiedlicher Größe (PM10 und PM2,5) und die Anzahl Stunden Ozonbelastung über dem Grenzwert. Die Modellwerte liegen als fein aufgelöste Rasterkarte $(200 \mathrm{~m} \times 200 \mathrm{~m}$ für Stickstoffdioxid und Feinstaub, $250 \mathrm{~m} \times 250 \mathrm{~m}$ für Ozon) vor. Die Werte für Stickstoffdioxid, Feinstaub und Ozon basieren auf Messwerten und Modellrechnungen aus dem Jahr 2000. Diese Werte sind aber relativ robust über längere Zeiträume. Wir haben die Zusammenhänge mit aus Szenarien geschätzten Werten für 2006 berechnet. Die Korrelationen liegen bei 0,9 und höher. Genaue Angaben zu den Modellrechnungen zur Ermittlung von Stickstoffdioxid, Feinstaub und Ozon sind in Anhang 1 zu finden.

Verkehrslärm am Tag und in der Nacht in Dezibel wird ebenfalls per Modellrechnung für jeweils $10 \mathrm{~m}$ große, quadratische Flächenstücke bestimmt. In SonBase, der GIS-Lärmdatenbank der Schweiz, wird auf Basis von Daten zur Verkehrsmenge, Geschwindigkeit und anderen Grundlagendaten die Emissionen der Quellen berechnet. Anschließend wird unter Einbezug des digitalen Höhenmodells in der Lärmberechnungssoftware CadnA die Ausbreitungsdämpfung ermittelt und die Lärmimmission berechnet (vgl. BAFU 2009c). Die Dezibel-Skala ist logarithmisch. Eine Erhöhung um drei Einheiten, z. B. von 50 auf 53, bedeutet, dass sich der physikalische Schalldruck verdoppelt. Jeder Wert eines Indikators ist ein Zahlentripel, bestehend aus dem Messwert und zwei Koordinatenwerten für den Ort. Aus den Befragungsdaten des Umweltsurveys kann die gleiche Datenstruktur erzeugt werden. Dazu müssen die Adressen der Haushalte in Koordinatenwerte umge-

1 Obligatorische Schule, Haushaltslehrjahr, Anlehre: 9 Jahre; Berufslehre, Vollzeitberufsschule: 12 Jahre; Berufsmaturität, Gymnasiale Matura, Lehrerkräfteseminart: 13 Jahre; Höhere Fachprüfung, Technikerschule: 14 Jahre; Fachhochschule, Technikum: 16 Jahre; Universitätsabschluss: 19 Jahre. 


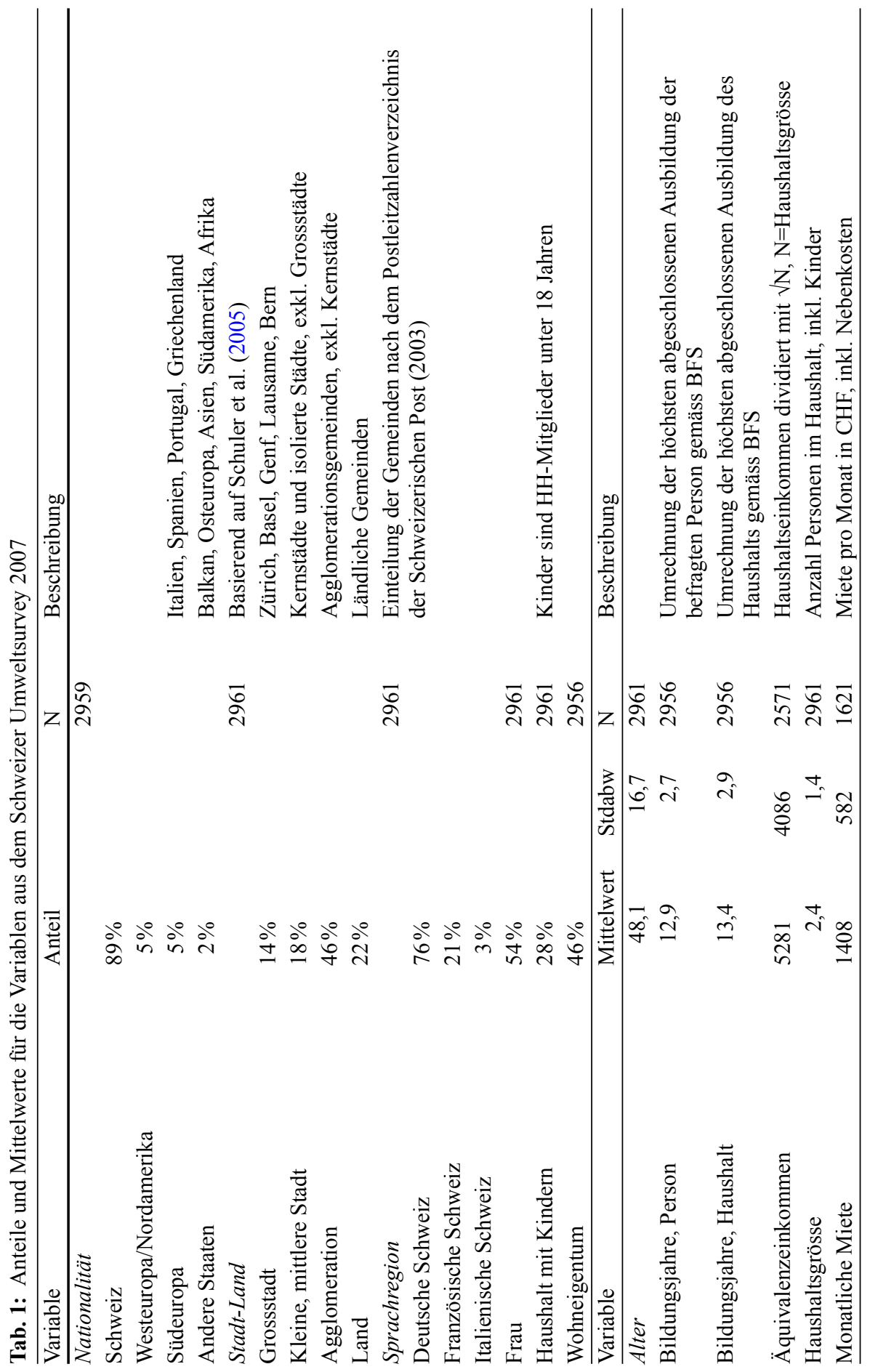




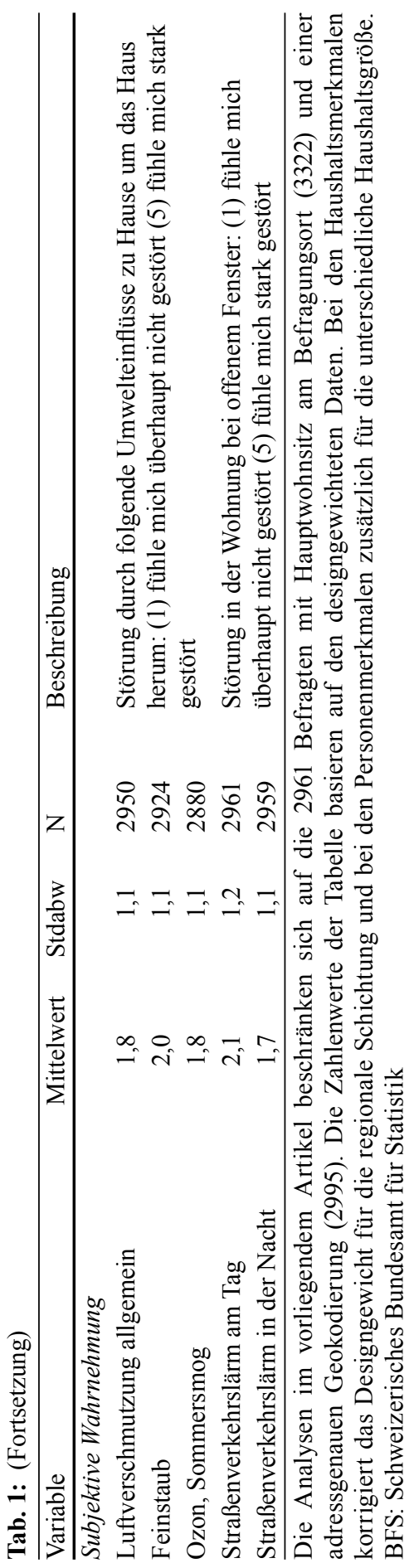


wandelt werden, d.h. die Haushalte werden geo-kodiert. ${ }^{2}$ Auch eine einzelne Ausprägung einer Variablen des Umweltsurveys wird dann zu einem Zahlentripel: Der Wert der Variablen plus zwei Koordinatenwerte für den Ort. Über die Koordinatenwerte können beide Datensätze verknüpft werden. Den einzelnen Befragten werden die Indikatoren der Luftverschmutzung und Lärmbelastung zugespielt. Aus Tab. 2 gehen die deskriptiven Werte der Umweltindikatoren hervor.

Luftverschmutzung (mit Ausnahme von Ozon in städtischen Gebieten, dazu weiter unten) und Lärmbelastung sind in größeren Städten und im stark vom Verkehr in Mitleidenschaft gezogenen Tessin besonders ausgeprägt. Einen Eindruck von der räumlichen Verteilung der Indikatoren der Umweltbelastung mit den Daten für unsere Stichprobe gibt Abb. 1.

\section{Indikatoren sozialer Schichtung und Umweltbelastung}

Wir berücksichtigen im ersten Schritt keine Drittvariablen. Uns interessiert zunächst nur, ob Bildung, Einkommen und Nationalität im Zusammenhang mit lokalen Umweltbelastungen stehen. Tabelle 3 enthält die Ergebnisse von bivariaten Korrelationen mit den Indikatoren der Umweltbelastung. Die Ergebnisse überraschen. Das Einkommen steht in keinem signifikanten Zusammenhang mit der Umweltbelastung, bei der Bildung findet man für Luftverschmutzung, ausgenommen Ozon, sogar einen signifikant positiven Zusammenhang.

Einzig die Mehrbelastung der Ausländer steht im Einklang mit den Hypothesen aus der Forschung über Umweltgerechtigkeit. Ausländer aus Südeuropa und aus anderen nichtwestlichen Staaten (Balkan, Osteuropa, Asien, Südamerika, Afrika) leben an Orten mit höherer Luftverschmutzung und ihre Wohnungen liegen an Straßen mit höherem Verkehrslärm. Beim Lärm ist die durchschnittliche Mehrbelastung der Südeuropäer (mit rund 2,1 Dezibel in der Nacht) ${ }^{3}$ und der Immigranten aus ,anderen Staaten“ (mit 2,8 Dezibel nachts) erheblich. Dabei ist aber zu bedenken, dass letztere Kategorie heterogen ist und nur rund $2 \%$ der Befragten enthält.

Wird die Größe des Wohnorts (wir verwenden vier Kategorien vom Land bis zur Großstadt) in der Regressionsgleichung berücksichtigt, vermindern sich die Schätzwerte für die Ausländerkategorien leicht. ${ }^{4}$ Ein Teil der höheren Belastung der Ausländer geht darauf zurück, dass diese in stärkerem Maße in Städten ansässig sind als Befragte mit Schweizer Pass. Allerdings sieht man auch, dass die Stadt-Land-Differenz mit Blick auf die Luftverschmutzung bedeutsamer ist als Nationalität und Einkommen (Tab. 4).

Zwar sind die Effekte des Einkommens auf die Indikatoren der Luftbelastung negativ und signifikant. Einkommensstärkere Haushalte scheinen demnach Umweltbelange bei

2 Für die Analysen in diesem Artikel werden die 2961 Personen mit Hauptwohnsitz (3 322) berücksichtigt, die aufgrund der Angaben aus der Befragung adressgenau geokodiert werden konnten (2995).

$3 \mathrm{Zu}$ den Mittelwerten für die einzelnen Kategorien der Nationalitäten siehe Tab. 7 im InternetAnhang.

4 Vgl. Tab. 7 und 10 im Internet-Anhang. 


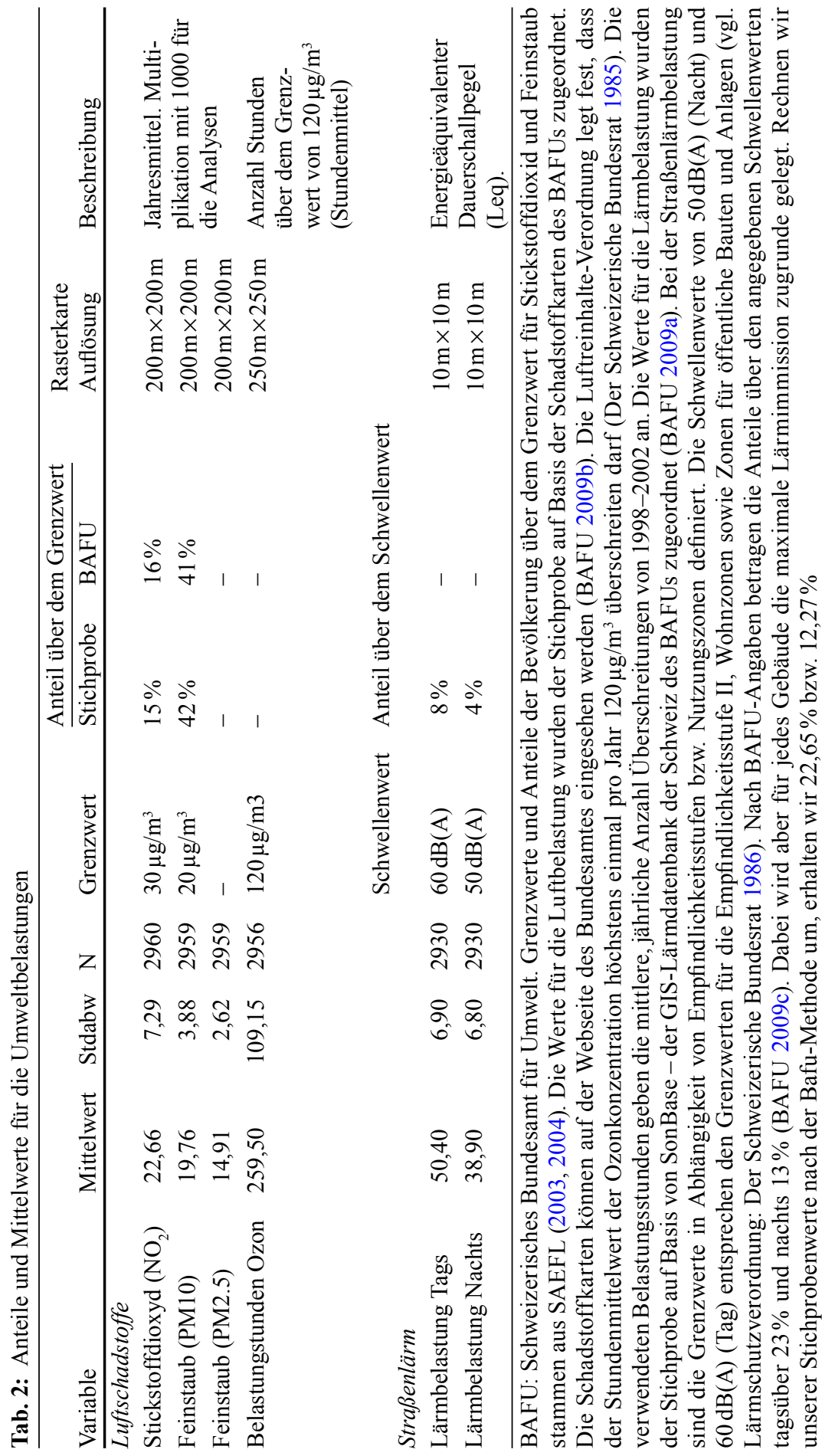




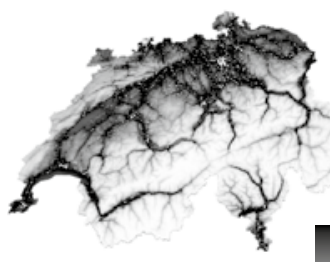

Stickstoffdioxid (NO2)
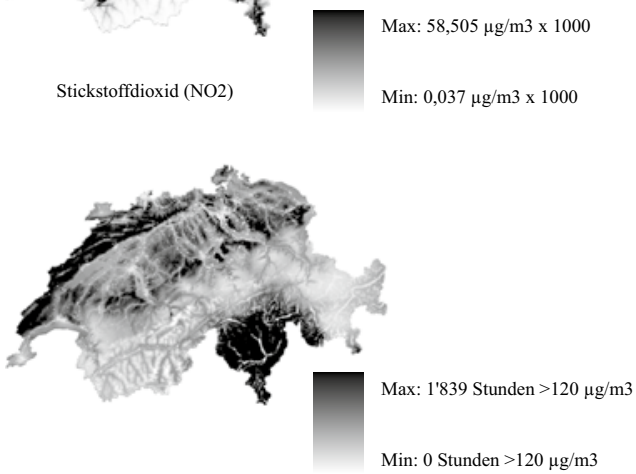

Bodennahes Ozon
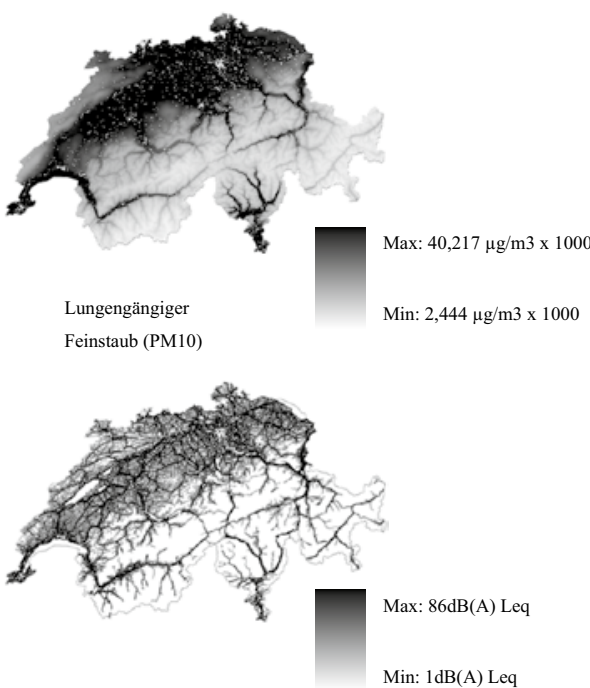

Straßenlärmbelastung Tag

Abb. 1: Räumliche Verteilung der Umweltbelastung. (Quellen: Luftbelastung: Schadstoffkarten Bundesamt für Umwelt (SAEFL 2003; 2004; BAFU 2009b). Straßenlärmbelastung: SonBase - Die GIS-Lärmdatenbank der Schweiz des Bundesamtes für Umwelt (BAFU 2009c). Helle Punkte: Wohnorte Befragte: Schweizer Umweltsurvey 2007 (Diekmann, Meyer 2008))

der Wohnortwahl nicht zu ignorieren. Dennoch ist das Ausmaß der Effekte sehr gering. Der Sozialgradient des Einkommens ist im Vergleich zur Stadt-Land-Differenz sehr schwach ausgeprägt.

Nehmen wir die Feinstaubbelastung (PM10) als Beispiel. Sie ist erwartungsgemäß in Großstädten mit plus 8,5 Einheiten im Vergleich zum Land am höchsten. Diese Differenz ist $120 \mathrm{Mal}$ größer als der Einkommenseffekt von $-0,07$ bezogen auf ein Mehreinkommen von 1000 Schweizer Franken monatlich (Tab.4).

Tab. 3: Bivariate Korrelationen von Indikatoren des Sozialstatus und Nationalität mit der Umweltbelastung

\begin{tabular}{llllllll}
\hline & \multicolumn{2}{l}{ Luftbelastung } & & & \multicolumn{2}{l}{ Straßenlärm } \\
\cline { 2 - 4 } & $\mathrm{NO}_{2}$ & PM10 & PM2.5 & Ozon & & Tag & Nacht \\
\hline Schweizer/In & Ref. & Ref. & Ref. & Ref. & & Ref. & Ref. \\
Ausländer & $0,10^{* *}$ & $0,08^{* *}$ & $0,08^{* *}$ & $-0,000$ & & $0,07^{* *}$ & $0,08^{* *}$ \\
& 0,000 & 0,000 & 0,000 & 0,812 & & 0,000 & 0,000 \\
Bildungsjahre HH (BFS & $0,10^{* *}$ & $0,08^{* *}$ & $0,08^{* *}$ & $-0,06^{* *}$ & & 0,010 & $-0,020$ \\
2007, in Zehner) & 0,000 & 0,000 & 0,000 & 0,001 & & 0,663 & 0,404 \\
Äquivalenzeinkommen & $-0,04^{+}$ & $-0,03^{+}$ & $-0,03^{+}$ & $-0,020$ & & $-0,020$ & $-0,030$ \\
(mon. in Tsd.) & 0,074 & 0,085 & 0,080 & 0,385 & & 0,302 & 0,194 \\
Durchschnittliche Anzahl & 2828 & 2827 & 2827 & 2824 & & 2801 & 2801 \\
Fälle & & & & & & \\
\hline
\end{tabular}

$\mathrm{p}$-Werte unter den bivariaten Korrelationskoeffizienten (keine Kontrollvariablen), ${ }^{+} \mathrm{p}<0,10$, ${ }^{*} \mathrm{p}<0,05,{ }^{* *} \mathrm{p}<0,01$ 
Tab. 4: OLS Regressionen mit der Umweltbelastung in Abhängigkeit von der Nationalität, Indikatoren des Sozialstatus und der Wohnortgröße

\begin{tabular}{|c|c|c|c|c|c|c|}
\hline & \multicolumn{4}{|c|}{ Luftbelastung } & \multicolumn{2}{|c|}{ Straßenlärm } \\
\hline & $\mathrm{NO}_{2}$ & PM10 & PM2.5 & Ozon & Tag & Nacht \\
\hline Schweizer/In & Ref. & Ref. & Ref. & Ref. & Ref. & Ref. \\
\hline $\begin{array}{l}\text { Westeuropa, } \\
\text { Nordamerika }\end{array}$ & $\begin{array}{l}-0,37 \\
(-0,73)\end{array}$ & $\begin{array}{l}-0,18 \\
(-0,60)\end{array}$ & $\begin{array}{l}-0,10 \\
(-0,47)\end{array}$ & $\begin{array}{l}2,77 \\
(0,28)\end{array}$ & $\begin{array}{l}0,54 \\
(0,90)\end{array}$ & $\begin{array}{l}0,62 \\
(-1,02)\end{array}$ \\
\hline Südeuropa & $\begin{array}{l}2,28 * * \\
(4,10)\end{array}$ & $\begin{array}{l}1,44 * * \\
(4,35)\end{array}$ & $\begin{array}{l}0,91 * * \\
(4,01)\end{array}$ & $\begin{array}{l}18,82^{+} \\
(1,73)\end{array}$ & $\begin{array}{l}1,48^{*} \\
(2,22)\end{array}$ & $\begin{array}{l}1,84 * * \\
(2,75)\end{array}$ \\
\hline Andere Staaten & $\begin{array}{l}3,20 * * \\
(4,16)\end{array}$ & $\begin{array}{l}1,72 * * \\
(3,74)\end{array}$ & $\begin{array}{l}1,05 * * \\
(3,33)\end{array}$ & $\begin{array}{l}-21,23 \\
(-1,41)\end{array}$ & $\begin{array}{l}1,89^{*} \\
(2,01)\end{array}$ & $\begin{array}{l}2,03 * \\
(2,14)\end{array}$ \\
\hline $\begin{array}{l}\text { Bildungsjahre } \mathrm{HH} \\
\text { (BFS 2007, in } \\
\text { Zehner) }\end{array}$ & $\begin{array}{l}0,27 \\
(0,68)\end{array}$ & $\begin{array}{l}0,04 \\
(0,17)\end{array}$ & $\begin{array}{l}0,02 \\
(0,13)\end{array}$ & $\begin{array}{l}0,74 \\
(0,10)\end{array}$ & $\begin{array}{l}-0,54 \\
(-1,13)\end{array}$ & $\begin{array}{l}-0,76 \\
(-1,58)\end{array}$ \\
\hline $\begin{array}{l}\text { Äquivalenzeinkom- } \\
\text { men (mon. in Tsd.) }\end{array}$ & $\begin{array}{l}-0,14^{* *} \\
(-4,88)\end{array}$ & $\begin{array}{l}-0,07^{* *} \\
(-4,28)\end{array}$ & $\begin{array}{l}-0,05 * * \\
(-4,15)\end{array}$ & $\begin{array}{l}-0,05 \\
(-0,08)\end{array}$ & $\begin{array}{l}-0,04 \\
(-1,13)\end{array}$ & $\begin{array}{l}-0,04 \\
(-1,19)\end{array}$ \\
\hline Ländliches Gebiet & Ref. & Ref. & Ref. & Ref. & Ref. & Ref. \\
\hline Agglomeration & $\begin{array}{l}6,55 * * \\
(21,47)\end{array}$ & $\begin{array}{l}3,36^{* *} \\
(18,52)\end{array}$ & $\begin{array}{l}2,29 * * \\
(18,41)\end{array}$ & $\begin{array}{l}-22,83^{* *} \\
(-3,82)\end{array}$ & $\begin{array}{l}2,30 * * \\
(6,33)\end{array}$ & $\begin{array}{l}2,61 * * \\
(7,16)\end{array}$ \\
\hline $\begin{array}{l}\text { Kleine od. Mittlere } \\
\text { Stadt }\end{array}$ & $\begin{array}{l}8,33 * * \\
(22,49)\end{array}$ & $\begin{array}{l}2,90 * * \\
(13,13)\end{array}$ & $\begin{array}{l}1,91 * * \\
(12,61)\end{array}$ & $\begin{array}{l}-54,37 * * \\
(-7,50)\end{array}$ & $\begin{array}{l}5,03 * * \\
(11,37)\end{array}$ & $\begin{array}{l}4,00 * * \\
(8,99)\end{array}$ \\
\hline Großstadt & $\begin{array}{l}16,91^{* *} \\
(50,44)\end{array}$ & $\begin{array}{l}8,50 * * \\
(42,58)\end{array}$ & $\begin{array}{l}5,45^{* *} \\
(39,78)\end{array}$ & $\begin{array}{l}-139,77 * * \\
(-21,30)\end{array}$ & $\begin{array}{l}5,37^{* *} \\
(13,40)\end{array}$ & $\begin{array}{l}4,21 * * \\
(10,45)\end{array}$ \\
\hline Konstante & $\begin{array}{l}16,42 * * \\
(29,59)\end{array}$ & $\begin{array}{l}17,24 * * \\
(52,13)\end{array}$ & $\begin{array}{l}13,27 * * \\
(58,52)\end{array}$ & $\begin{array}{l}303,35^{* *} \\
(27,92)\end{array}$ & $\begin{array}{l}48,52 * * \\
(73,02)\end{array}$ & $\begin{array}{l}37,50^{* *} \\
(56,12)\end{array}$ \\
\hline Korr. R-Quadrat & 0,526 & 0,446 & 0,409 & 0,195 & 0,086 & 0,052 \\
\hline Anzahl Fälle & 2569 & 2568 & 2568 & 2565 & 2546 & 2546 \\
\hline
\end{tabular}

t-Werte in Klammern, ${ }^{+} \mathrm{p}<0,10,{ }^{*} \mathrm{p}<0,05,{ }^{*} \mathrm{p}<0,01$

Im Unterschied zu Stickstoffdioxid und Feinstaub sind die Ozonwerte paradoxerweise in urbanen Gebieten geringer als auf dem Land. Dies, obwohl auch hier der Verkehr ursächlich für die Luftverschmutzung ist. Der Grund ist, dass die gleichen Abgase, die tagsüber zur Erzeugung des Ozons beigetragen haben, nach Anbruch der Dunkelheit einen Teil des Ozons in städtischen Gebieten zersetzen (dazu genauer im Anhang). Auf dem (stadtnahen) Land breitet sich das in den Städten produzierte Ozon aus, ohne dass hier Gegenkräfte wirksam werden. Deshalb misst man auf dem Land höhere Ozonwerte. Dies ist der Grund, dass wir bei Ozon das umgekehrte Vorzeichen finden im Vergleich zu allen anderen Indikatoren der Luftqualität.

Um ein genaueres Bild zu erhalten, „kontrollieren“ wir in multivariaten Analysen weitere soziodemografische Variablen, die mutmaßlich mit der Umweltbelastung am Wohnort zusammenhängen. Wer Wohneigentum erwirbt, ist in der Regel wohlhabender und wird vermutlich auch darauf achten, dass sich die Immobilie an Orten befindet, die weniger umweltbelastet sind. Zudem stellt sich die Frage, ob diese und weitere Merkmale die Mehrbelastung der Ausländer eventuell ,erklären“ können. Wir berücksichtigen zusätzlich die Haushaltsmerkmale Sprachregion, Wohneigentum und Haushaltsgröße und kontrollieren für die Befragtenmerkmale Alter und Geschlecht. Über die Ergebnisse 
der Regressionsanalysen für die sechs Indikatoren der Umweltbelastung informiert Tab. 6 im Anhang.

Auch unter Kontrolle weiterer soziodemografischer Merkmale ist eine signifikant erhöhte Umweltbelastung der Ausländer nachweisbar. Das Einkommen hat im Einklang mit den Erwartungen einen signifikant negativen Effekt. Die Größenordnung des Einkommenseffekts und die Mehrbelastung der Ausländer bei der Luftverschmutzung sind aber geringer als die Stadt-Land-Differenz. Wohneigentümer und Mitglieder größerer Haushalte leben an Orten mit geringerer Luft- und Lärmbelastung. Auffallend ist auch die höhere Luftverschmutzung in der italienischen Schweiz.

Bei der Bildung sind die Ergebnisse ambivalent. Je nach berücksichtigten Kovariaten finden wir nicht signifikante Werte der Koeffizienten (Tab.4) und sogar, im Gegensatz zu den Erwartungen, signifikant-positive Zusammenhänge mit den Indikatoren der Luftbelastung (Tab. 6 im Anhang). Die absoluten Unterschiede sind allerdings sehr gering. Eine Differenz von zehn Bildungsjahren entspricht nur einem sehr kleinen Bruchteil der Mehrbelastung in der Großstadt gegenüber dem Land.

Fügen wir noch als weiteres Merkmal die im GIS berechnete Distanz der Wohnung zu Einkaufsmöglichkeiten, Post und öffentlichem Verkehr hinzu, schwächen sich die Effekte bei den Ausländern leicht ab. Die „Distanz“ weist ein signifikant negatives Vorzeichen bei der Luft- und Lärmbelastung auf, jedoch mit Ausnahme von Ozon. Bei letzterem Schadstoff wiederum ist der Koeffizient signifikant positiv. Dies Muster ist leicht erklärbar. Bei größerer Distanz liegt die Wohnung vermutlich in ruhigeren Seitenstraßen abseits der Zentren urbaner Betriebsamkeit oder der Gemeindekerne (Tab. 16 im Internetanhang). ${ }^{5}$

Nun hängt die Wahl eines Wohnorts sowohl von den Vorlieben (Präferenzen) eines Haushalts und von seinen Möglichkeiten (Opportunitäten) ab. Bei gleichem Einkommen wird Haushalt A z. B. eine ruhige Wohnlage, jedoch mit ungünstiger Verkehrsanbindung bevorzugen, während für Haushalt B störender Lärm weniger wichtig ist als Einkaufsmöglichkeiten und Mobilität. Kontrolliert man in der Regressionsschätzung für Einkommen, so könnte man argumentieren, spiegeln verbleibende Umweltbelastungen eben Präferenzen wider. Wir sind allerdings nicht der Auffassung, dass Präferenzen und Opportunitäten so einfach zu separieren sind. Die Lage der Wohnung hängt von vielen anderen Merkmalen ab. Dazu zählen u. a. die Suchdauer und Suchkosten, soziale Netzwerke, Ort und Art beruflicher Tätigkeit und eine mögliche Diskriminierung auf dem Wohnungsmarkt. Außerdem sind in Mehrpersonenhaushalten oft nicht alle Mitglieder an der Entscheidung über den Wohnort beteiligt. Wenn die Eltern z. B. die Umweltbelastung ignorieren, sind die Kinder unfreiwillig mitbetroffen und eventuell höheren Gesundheitsrisiken ausgesetzt. Wir untersuchen, ob soziale Gruppen mehr oder minder von Umweltbelastungen betroffen sind. In welchem Ausmaß unterschiedliche Präferenzen dabei wirksam sind, ist eine müßige Spekulation und mittels der Surveydaten kaum beantwortbar.

\section{Subjektive Wahrnehmung der Umweltbelastung}

In der „Risikogesellschaft“ von Ulrich Beck (1986) wird als Besonderheit der neuen Risiken genannt, dass diese nicht sinnlich wahrnehmbar seien. Das mag auf Radioaktivität

5 Internetanhang: http://www.socio.ethz.ch/research/umweltsurvey/umweltsurvey2007/publications. 
oder Treibhausgase zutreffen. Andere Risiken sind aber sehr wohl menschlichen Sinnen zugänglich. Die Wahrnehmung von Luftverschmutzung und Lärm haben wir im telefonischen Interview mit einer fünfstufigen und Lärm in der schriftlichen Befragung zudem mit einer zehnstufigen Ratingskala erhoben. Die Korrelationen der beiden Erhebungen betragen für den Straßenverkehrslärm am Tag 0,71 und in der Nacht 0,66. Wir stützen uns auch für Lärm auf die etwas umfangreicheren Daten der telefonischen Befragung.

Eine Regressionsanalyse mit den Bewertungen als abhängige Variable, soziodemografischen Merkmalen und der objektiven Belastung als unabhängige Variable gibt Auskunft über die Zusammenhänge. Es zeigt sich, dass Ausländer, Stadtbewohner und Befragte in Haushalten mit geringerem Einkommen der Tendenz nach stärkere Umweltbelastungen wahrnehmen (Tab.5). Gleiches gilt für Befragte in der italienischen und

Tab. 5: OLS Regression subjektive Wahrnehmung der Umweltbelastung

\begin{tabular}{|c|c|c|c|c|c|c|}
\hline & \multicolumn{4}{|l|}{ Luftbelastung } & \multicolumn{2}{|c|}{ Straßenlärm } \\
\hline & (1) Allgemein & (2) Feinstaub & (3) Feinstaub & (4) Ozon & (5) Tag & (6) Nacht \\
\hline Objektive Belastung & $\begin{array}{l}0,04 * * \\
(9,61)\end{array}$ & $\begin{array}{l}0,06^{* *} \\
(6,78)\end{array}$ & $\begin{array}{l}0,09 * * \\
(6,30)\end{array}$ & $\begin{array}{l}-0,08 * * \\
(-3,33)\end{array}$ & $\begin{array}{l}0,05^{* *} \\
(14,61)\end{array}$ & $\begin{array}{l}0,03 * * \\
(10,33)\end{array}$ \\
\hline Schweizer/In & Ref. & Ref. & Ref. & Ref. & Ref. & Ref. \\
\hline $\begin{array}{l}\text { Westeuropa, } \\
\text { Nordamerika }\end{array}$ & $\begin{array}{l}-0,02 \\
(-0,20)\end{array}$ & $\begin{array}{l}-0,07 \\
(-0,66)\end{array}$ & $\begin{array}{l}-0,07 \\
(-0,70)\end{array}$ & $\begin{array}{l}-0,16 \\
(-1,57)\end{array}$ & $\begin{array}{l}0,27 * \\
(2,44)\end{array}$ & $\begin{array}{l}0,05 \\
(0,52)\end{array}$ \\
\hline Südeuropa & $\begin{array}{l}0,12 \\
(1,06)\end{array}$ & $\begin{array}{l}0,32 * * \\
(2,67)\end{array}$ & $\begin{array}{l}0,33 * * \\
(2,74)\end{array}$ & $\begin{array}{l}-0,02 \\
(-0,15)\end{array}$ & $\begin{array}{l}-0,05 \\
(-0,40)\end{array}$ & $\begin{array}{l}-0,1 \\
(-0,93)\end{array}$ \\
\hline Andere Staaten & $\begin{array}{l}0,04 \\
(0,27)\end{array}$ & $\begin{array}{l}0,28^{+} \\
(1,68)\end{array}$ & $\begin{array}{l}0,29^{+} \\
(1,76)\end{array}$ & $\begin{array}{l}0,29^{+} \\
(1,84)\end{array}$ & $\begin{array}{l}0,43^{*} \\
(2,47)\end{array}$ & $\begin{array}{l}0,35^{*} \\
(2,3)\end{array}$ \\
\hline $\begin{array}{l}\text { Bildungsjahre (BFS } \\
2007 \text {, in Zehner) }\end{array}$ & $\begin{array}{l}0,15^{+} \\
(1,74)\end{array}$ & $\begin{array}{l}0,07 \\
(0,76)\end{array}$ & $\begin{array}{l}0,07 \\
(0,78)\end{array}$ & $\begin{array}{l}0,15^{+} \\
(1,7)\end{array}$ & $\begin{array}{l}0,14 \\
(1,5)\end{array}$ & $\begin{array}{l}0,05 \\
(0,58)\end{array}$ \\
\hline $\begin{array}{l}\text { Äquivalenzeinkom- } \\
\text { men (mon. in Tsd.) }\end{array}$ & $\begin{array}{l}-0,01^{*} \\
(2,41)\end{array}$ & $\begin{array}{l}-0,01^{+} \\
(-1,65)\end{array}$ & $\begin{array}{l}-0,01^{+} \\
(-1,69)\end{array}$ & $\begin{array}{l}-0,01^{*} \\
(-2,19)\end{array}$ & $\begin{array}{l}-0,01 \\
(-1,43)\end{array}$ & $\begin{array}{l}-0,01 \\
(-1,45)\end{array}$ \\
\hline Ländliches Gebiet & Ref. & Ref. & Ref. & Ref. & Ref. & Ref. \\
\hline Agglomeration & $\begin{array}{l}-0,01 \\
(-0,11)\end{array}$ & $\begin{array}{l}0,12^{+} \\
(1,67)\end{array}$ & $\begin{array}{l}0,12^{+} \\
(1,78)\end{array}$ & $\begin{array}{l}0,25^{* *} \\
(3,96)\end{array}$ & $\begin{array}{l}0,1 \\
(1,43)\end{array}$ & $\begin{array}{l}0,10^{+} \\
(1,69)\end{array}$ \\
\hline $\begin{array}{l}\text { Kleine od. Mittlere } \\
\text { Stadt }\end{array}$ & $\begin{array}{l}0,05 \\
(0,64)\end{array}$ & $\begin{array}{l}0,22 * * \\
(2,73)\end{array}$ & $\begin{array}{l}0,23 * * \\
(2,87)\end{array}$ & $\begin{array}{l}0,31 * * \\
(3,9)\end{array}$ & $\begin{array}{l}0,06 \\
(0,67)\end{array}$ & $\begin{array}{l}0,17^{*} \\
(2,31)\end{array}$ \\
\hline Großstadt & $\begin{array}{l}0,12 \\
(1,26)\end{array}$ & $\begin{array}{l}0,25^{*} \\
(2,32)\end{array}$ & $\begin{array}{l}0,29 * * \\
(2,8)\end{array}$ & $\begin{array}{l}0,65^{* *} \\
(8,28)\end{array}$ & $\begin{array}{l}0,31 * * \\
(3,84)\end{array}$ & $\begin{array}{l}0,33 * * \\
(4,76)\end{array}$ \\
\hline Konstante & $\begin{array}{l}0,78 * * \\
(4,32)\end{array}$ & $\begin{array}{l}0,363 \\
(1,55)\end{array}$ & $\begin{array}{l}0,28 \\
(1,09)\end{array}$ & $\begin{array}{l}1,60 * * \\
(9,08)\end{array}$ & $\begin{array}{l}-0,67 * * \\
(-2,60)\end{array}$ & $\begin{array}{l}0,3 \\
(1,5)\end{array}$ \\
\hline Korr. R-Quadrat & 0,106 & 0,092 & 0,09 & 0,086 & 0,117 & 0,071 \\
\hline Anzahl Fälle & 2560 & 2542 & 2542 & 2501 & 2544 & 2543 \\
\hline
\end{tabular}

t-Werte in Klammern, ${ }^{+} \mathrm{p}<0,10,{ }^{*} \mathrm{p}<0,05,{ }^{* *} \mathrm{p}<0,01$. Weitere Kovariaten sind Sprachregion, Eigentümer, Haushaltsgröße, Alter und Geschlecht. Die vollständigen Tabellen sind im InternetAnhang. „Allgemein“ steht für Störung durch Luftverschmutzung allgemein. Messungen der objektiven Belastung: 1) $\mathrm{NO}_{2}$, 2) PM10, 3) PM2.5, 4) Ozon: Anzahl Stunden über dem Grenzwert, 5) Straßenlärm tagsüber, 6) Straßenlärm nachts 
französischen Schweiz und für Personen ohne Wohneigentum. ${ }^{6}$ Lassen wir zunächst einmal Ozon bei Seite, so ist weiterhin erkennbar, dass die objektive Belastung konsistente, signifikant positive Effekte auf die Wahrnehmung ausübt. Die „objektiven“ Zusammenhänge werden der Richtung der Effekte nach in der subjektiven Wahrnehmung recht gut widergespiegelt. Allerdings ist die erklärte Varianz relativ gering, auch im Vergleich zur Modellgüte in den objektiven Regressionen (vgl. R-Quadrate der Tab. 4 und 5). Die subjektive Bewertung ist im Einzelfall kein Ersatz für eine objektive Messung.

Die Wahrnehmung von Ozonsmog ist die große Ausnahme. Wir hatten schon weiter oben festgehalten, dass in den Städten paradoxerweise weniger Ozon gemessen wird als auf dem Land. Dies kommt in der individuellen Bewertung nicht zum Ausdruck. Im Gegenteil: Die physikalische Messung hat ein negatives Vorzeichen, der Schätzwert ist signifikant.

Je höher die tatsächliche Ozonbelastung, desto optimistischer ist die subjektive Wahrnehmung. Wer an wenig verkehrsreichen Orten lebt und sich bezüglich Stickstoffdioxid und Feinstaub einer besseren Luftqualität erfreut, überträgt diese Wahrnehmung nach Art eines Halo-Effekts auch und zu Unrecht auf das Niveau der Ozonbelastung. ${ }^{7}$ Nach gleicher Logik ist das Vorzeichen für urbane Gebiete in der Regressionsgleichung für Ozon-Smog positiv. Wer in städtischen Gebieten lebt, nimmt subjektiv mehr Ozon-Smog wahr, obwohl faktisch der Grenzwert auf dem stadtnahen Land häufiger übertroffen wird als in urbanen Zonen.

\section{Diskussion und Ausblick}

Unsere Untersuchung mit den Befragungsdaten des Schweizer Umweltsurveys und den geo-referenzierten Messungen der Umweltbelastung ergibt ein differenziertes Bild. Benachteiligungen im Sinne der Forschung über Umweltgerechtigkeit zeigen sich deutlich bei Ausländern, allerdings nur bei bestimmten Nationalitäten. Während Westeuropäer keine höheren Belastungen ertragen müssen als Inländer, sind Südeuropäer und Haushalte mit Bewohnern aus den Balkanstaaten, Osteuropa und aus außereuropäischen Ländern durchaus mit erheblichen Umweltbeeinträchtigungen konfrontiert. Überraschend und im Gegensatz zu den Erwartungen stehen Indikatoren des Sozialstatus wie Einkommen und Bildung auf bivariater Ebene nicht in einem signifikanten Zusammenhang mit den hier betrachteten Umweltindikatoren. Allerdings sind die Zusammenhänge komplexer. In der multivariaten Analyse zeigt sich zwar ein signifikanter Effekt des Einkommens, das Ausmaß des Effekts ist aber relativ schwach. Weiterhin erklärt das Einkommen nicht die erhöhte Belastung der erwähnten Ausländergruppen. In stärkerem Maße sinkt die Umweltlast, wenn Wohneigentum erworben wird. Am stärksten ausgeprägt ist aber die Stadt-Land-Differenz. Wer aufs Land zieht, reduziert die Umweltbelastung wesentlich. Um ein Gefühl für die Größenordnung zu geben: Bei Stickstoffdioxid haben Eigentümer

6 Ergebnisse hier nicht dargestellt. Siehe dazu Tab. 13 im Internet-Anhang.

7 Die Frage bezieht sich allerdings nicht nur auf Ozon, sondern auf Ozon und Sommersmog. Der Fragetext lautet: „Wie stark fühlen Sie sich bei Ihnen um das Haus herum durch folgende Umwelteinflüsse gestört? Bodennahes Ozon, also Sommersmog bei Ihnen um das Haus herum.“ 
im Durchschnitt eine um 2 Einheiten verminderte Belastung im Vergleich zu Mietern (bei Kontrolle weiterer sozialdemographischer Merkmale; vgl. Tab. 6 im Anhang 2). Der Unterschied Großstadt versus Land macht dagegen 16 Einheiten aus, also ein Faktor 8 gegenüber dem Effekt von Wohneigentum. Ausgleichend kommt die paradoxe Wirkung von Ozonsmog hinzu, dessen Grenzwert auf dem Land häufiger überschritten wird als in den verkehrsreichen Städten. Die Zusammenhänge soziodemografischer Merkmale mit der subjektiven Wahrnehmung von Lärm und Luftverschmutzung entsprechen weitgehend den „objektiven“ Zusammenhängen. Nur beim Ozonsmog ist es anders. Wer stärker betroffen ist, berichtet eher geringere Belastungen. Diese „Ozon-Täuschung“ erklären wir durch eine Art Halo-Effekt.

Nimmt man Einkommen und Bildung als Kriterien, ist der Schweizer Smog ,demokratisch" verteilt. Berücksichtigt man Wohneigentum und die Nationalität, sind dagegen Mieter und Ausländer von Umweltrisiken stärker betroffen als Eigentümer und Inländer. Ist der flache Sozialgradient beim Einkommen ein Schweizer Sonderfall? Die Schweiz ist föderaler, kleinräumiger, ohne ausgeprägte Großstädte und erkennbare Slumgebiete und auch das Ausmaß der Segregation von Wohngebieten dürfte geringer ausfallen als in den Ballungsgebieten anderer Staaten. Hinzu kommen das relativ hohe Durchschnittseinkommen und die ausgebaute Infrastruktur in den urbanen Gebieten der Schweiz. Ob sich die geringe Korrelation mit dem Haushaltseinkommen als eine Besonderheit der Schweiz herausstellt, etwa im Unterschied zu den USA oder anderen Ländern, ist eine Frage, die nur empirisch beantwortet werden kann. Vergleichende Studien mit geo-referenzierten Daten könnten hierüber Aufschluss geben.

In methodischer Hinsicht demonstriert unsere Arbeit, dass es für die soziologische Forschung von großem Nutzen sein kann, geo-referenzierte Daten zu erschließen und mit Individualdaten aus Umfragen zu verbinden. Effekte der räumlichen Umgebung auf Einstellungen, Wahrnehmungen, Befindlichkeiten und andere Merkmale von Personen und Haushalten können auf diese Weise genauer untersucht werden. Zum räumlichen Kontext zählen die Nähe zu Schulen, Kindergärten, Verkehrsmitteln, Arbeitsstätten, Einkaufsmöglichkeiten, die Art der Bebauung, die soziale Zusammensetzung von Nachbarschaften u. a.m. Viele dieser Merkmale und zahlreiche weitere sind geo-referenziert und liegen in entsprechenden Registern vor. Untersuchungen über die Aufnahme einer Erwerbstätigkeit, über Bildungsentscheidungen oder zur Fertilität, um nur einige Beispiele zu nennen, können von der Nutzung geo-referenzierter Daten nur profitieren.

Wie bei jeder Studie muss man aber auch gewisse Grenzen der Aussagekraft durch die Spezifika der Datenerhebung und Messung beachten. In unserer Untersuchung haben wir sechs ausgewählte Indikatoren von Lärm und Luftverschmutzung verwendet, die sämtlich über Lärm und Luftqualität außerhalb der Wohnung informieren. Die Indikatoren messen so zu sagen die öffentliche Umweltbelastung, nicht aber ,private“ Belastungen in der Wohnung. Über Wohngifte, Schimmel und vor allem Zigarettenrauch in der Wohnung werden keine Aussagen gemacht. Hier sind aber durchaus stärkere Zusammenhänge mit der Sozialschicht zu erwarten (Braun-Fahrländer 2004; Becker et al. 2008). Schließlich kann man sich gegen Umweltbelastungen, insbesondere gegenüber Lärm, durch geeignete bauliche Maßnahmen, wie schallschützende Fenster, abschirmen. Wer über größere Wohnungen verfügt, kann zudem seine Aktivitäten in ruhigere, etwa hofseitig gelegene Wohnbereiche verlegen. Bei gleichen Lärmimmissionen (gemessen an der Außenwand) 
haben Haushalte mit größeren Einkommensressourcen mehr Gestaltungsmöglichkeiten, um lästigem Außenlärm zu entkommen. Allerdings finden wir mit unseren Daten keinen Zusammenhang zwischen dem Einbau von Lärmschutzfenstern und dem Einkommen. Ein weiterer Diskussionspunkt ist die Mehrbelastung der Haushalte von Ausländern. In der Stichprobe sind nur Ausländerhaushalte von Befragten, mit denen das Interview in einer der Landessprachen geführt werden konnte. Das heißt aber, dass gerade gut integrierte Ausländer mit höherer Wahrscheinlichkeit in der Stichprobe enthalten sind. Die Verzerrung der Ausländerstichprobe hat vermutlich zur Folge, dass die Umweltbelastung der Ausländerhaushalte eher unterschätzt wird. Stichproben aus Gemeinderegistern kombiniert mit geo-referenzierten Daten wären erforderlich, um das Ausmaß einer möglichen Verzerrung abzuschätzen.

\section{Anhang 1: Messung der Luftschadstoffe (Thomas Künzle, Meteotest)}

\section{Stickstoffdioxid und Feinstaub}

Die Ausbreitungsmodellierung für Stickstoffdioxid und Feinstaub erfolgte auf der Basis der Emissionen mit Transferfunktionen. Diese Funktionen stützen sich auf stündliche meteorologische Daten eines repräsentativen Jahres. Die Verwendung mehrerer Transferfunktionen ermöglicht es, die verschiedenen Eigenschaften der Emittentengruppen (Emissionshöhe, Zeitreihen der Emissionsstärke) und die Ausbreitungsbedingungen abzubilden (z.B. werden in Alpentälern die Transferfunktionen in Hauptwindrichtung ausgerichtet) (SAEFL 2003; 2004).

\section{Ozonbelastung}

Für die Berechnung der Anzahl Stunden Ozonbelastung über $120 \mu \mathrm{g} / \mathrm{m}^{3}$ wurden zuerst die Ozonmessungen der kantonalen Fachstellen für Luftreinhaltung und des nationale Beobachtungsnetz für Luftfremdstoffe (NABEL vgl. BAFU und EMPA 2009) der Jahre 1998-2002 gemittelt (Datengrundlage: BAFU und Cercl'Air 2009) und daraus die Karten erzeugt. Die aufbereiteten Messwerte wurden mithilfe eines geo-statistischen Ansatzes räumlich interpoliert. Dabei wurden zuerst statistische Beziehungen zwischen den gemessenen Ozonbelastungs-Indizes und flächendeckend im GIS vorhandenen Informationen gesucht. Anschließend wurden die punktförmigen Messungen auf Rasterbasis in die Fläche ausgedehnt, indem für jede Rasterzelle mit den ermittelten Regressionsgleichungen die Ozon-Indizes gerechnet wurden.

\section{Verteilung von Ozon in urbanen Gebieten und auf dem Land}

Das anthropogene Ozon in der Grundschicht der Atmosphäre ist ein sekundär gebildeter Stoff, der nicht direkt einer Schadstoffquelle entstammt. Es wird erst in der Atmosphäre durch photochemische Reaktionen aus so genannten Vorläuferschadstoffen, den Stickoxiden (NOx) und den flüchtigen organischen Verbindungen (VOC) gebildet. Zur Ozonbildung ist intensive Sonnenstrahlung notwendig, zudem begünstigen erhöhte Lufttemperaturen den Reaktionsablauf. Hohe Ozonkonzentrationen treten deshalb typischerweise während sommerlichen Hochdruckwetterlagen (Schönwetterperioden) auf. Die Ozonbildung in einem Hochdruckgebiet ist ein großräumiger Prozess, sie ist jedoch in der 
Umgebung von großen Agglomerationen besonders intensiv, da dort ein sehr reaktives Gemisch von Vorläuferschadstoffen, hauptsächlich aus dem Verkehr, vorliegt.

Der Text in Anhang 1 wurde von Thomas Künzle, Meteotest, verfasst.

\section{Anhang 2}

Tab. 6: OLS Regressionen soziale Verteilung Umweltbelastung, volles Modell

\begin{tabular}{|c|c|c|c|c|c|c|}
\hline & \multicolumn{4}{|c|}{ Luftbelastung } & \multicolumn{2}{|c|}{ Straßenlärm } \\
\hline & $\mathrm{NO}_{2}$ & PM10 & PM2.5 & Ozon & Tag & Nacht \\
\hline Schweizer/In & Ref. & Ref. & Ref. & Ref. & Ref. & Ref. \\
\hline $\begin{array}{l}\text { Westeuropa, } \\
\text { Nordamerika }\end{array}$ & $\begin{array}{l}-0,70 \\
(-1,45)\end{array}$ & $\begin{array}{l}-0,13 \\
(-0,58)\end{array}$ & $\begin{array}{l}-0,05 \\
(-0,33)\end{array}$ & $\begin{array}{l}7,68 \\
(0,94)\end{array}$ & $\begin{array}{l}0,21 \\
(0,35)\end{array}$ & $\begin{array}{l}0,29 \\
(0,49)\end{array}$ \\
\hline Südeuropa & $\begin{array}{l}1,31^{*} \\
(2,40)\end{array}$ & $\begin{array}{l}0,64 * \\
(2,49)\end{array}$ & $\begin{array}{l}0,35^{*} \\
(2,08)\end{array}$ & $\begin{array}{l}-14,15 \\
(-1,53)\end{array}$ & $\begin{array}{l}1,25^{+} \\
(1,84)\end{array}$ & $\begin{array}{l}1,54^{*} \\
(2,27)\end{array}$ \\
\hline Andere Staaten & $\begin{array}{l}2,36^{* *} \\
(3,15)\end{array}$ & $\begin{array}{l}1,13^{* *} \\
(3,18)\end{array}$ & $\begin{array}{l}0,66 * * \\
(2,80)\end{array}$ & $\begin{array}{l}-37,94 * * \\
(-2,99)\end{array}$ & $\begin{array}{l}1,51 \\
(1,59)\end{array}$ & $\begin{array}{l}1,59^{+} \\
(1,67)\end{array}$ \\
\hline $\begin{array}{l}\text { Bildungsjahre } \mathrm{HH} \\
\text { (BFS 2007, in } \\
\text { Zehner) }\end{array}$ & $\begin{array}{l}0,77^{*} \\
(1,97)\end{array}$ & $\begin{array}{l}0,48^{* *} \\
(2,62)\end{array}$ & $\begin{array}{l}0,33 * * \\
(2,70)\end{array}$ & $\begin{array}{l}-1,53 \\
(-0,23)\end{array}$ & $\begin{array}{l}-0,27 \\
(-0,55)\end{array}$ & $\begin{array}{l}-0,57 \\
(-1,17)\end{array}$ \\
\hline $\begin{array}{l}\text { Äquivalenzeinkom- } \\
\text { men (mon. in Tsd.) }\end{array}$ & $\begin{array}{l}-0,11^{* *} \\
(-3,89)\end{array}$ & $\begin{array}{l}-0,04 * * \\
(-3,22)\end{array}$ & $\begin{array}{l}-0,03^{* *} \\
(-3,17)\end{array}$ & $\begin{array}{l}1,09^{*} \\
(2,28)\end{array}$ & $\begin{array}{l}-0,03 \\
(-0,98)\end{array}$ & $\begin{array}{l}-0,04 \\
(-1,01)\end{array}$ \\
\hline Ländliches Gebiet & Ref. & Ref. & Ref. & Ref. & Ref. & Ref. \\
\hline Agglomeration & $\begin{array}{l}6,01 * * \\
(20,29)\end{array}$ & $\begin{array}{l}2,83 * * \\
(20,22)\end{array}$ & $\begin{array}{l}1,92 * * \\
(20,77)\end{array}$ & $\begin{array}{l}-32,51 * * \\
(-6,48)\end{array}$ & $\begin{array}{l}2,10 * * \\
(5,76)\end{array}$ & $\begin{array}{l}2,44 * * \\
(6,65)\end{array}$ \\
\hline $\begin{array}{l}\text { Kleine od. Mittlere } \\
\text { Stadt }\end{array}$ & $\begin{array}{l}7,25^{* *} \\
(19,86)\end{array}$ & $\begin{array}{l}2,07 * * \\
(12,00)\end{array}$ & $\begin{array}{l}1,34 * * \\
(11,78)\end{array}$ & $\begin{array}{l}-65,83 * * \\
(-10,65)\end{array}$ & $\begin{array}{l}4,48 * * \\
(9,95)\end{array}$ & $\begin{array}{l}3,50 * * \\
(7,72)\end{array}$ \\
\hline Großstadt & $\begin{array}{l}15,85^{* *} \\
(46,04)\end{array}$ & $\begin{array}{l}8,17 * * \\
(50,28)\end{array}$ & $\begin{array}{l}5,26 * * \\
(48,95)\end{array}$ & $\begin{array}{l}-124,19 * * \\
(-21,28)\end{array}$ & $\begin{array}{c}4,45^{* *} \\
(10,48)\end{array}$ & $\begin{array}{l}3,41^{* *} \\
(7,96)\end{array}$ \\
\hline Deutsche Schweiz & Ref. & Ref. & Ref. & Ref. & Ref. & Ref. \\
\hline $\begin{array}{l}\text { Französische } \\
\text { Schweiz }\end{array}$ & $\begin{array}{l}-0,17 \\
(-0,59)\end{array}$ & $\begin{array}{l}-2,37 * * \\
(-17,04)\end{array}$ & $\begin{array}{l}-1,70^{* *} \\
(-18,54)\end{array}$ & $\begin{array}{l}-10,00^{*} \\
(-2,01)\end{array}$ & $\begin{array}{l}0,43 \\
(1,18)\end{array}$ & $\begin{array}{l}0,55 \\
(1,49)\end{array}$ \\
\hline Italienische Schweiz & $\begin{array}{l}5,86^{* *} \\
(10,70)\end{array}$ & $\begin{array}{l}9,64 * * \\
(37,22)\end{array}$ & $\begin{array}{l}6,95 * * \\
(40,65)\end{array}$ & $\begin{array}{l}303,54 * * \\
(32,54)\end{array}$ & $\begin{array}{l}-0,61 \\
(-0,91)\end{array}$ & $\begin{array}{l}-0,41 \\
(-0,61)\end{array}$ \\
\hline Haushaltsgröße & $\begin{array}{l}-0,36^{* *} \\
(-4,01)\end{array}$ & $\begin{array}{l}-0,13^{* *} \\
(-3,05)\end{array}$ & $\begin{array}{l}-0,09^{* *} \\
(-3,27)\end{array}$ & $\begin{array}{l}5,13 * * \\
(3,33)\end{array}$ & $\begin{array}{l}-0,32 * * \\
(-2,83)\end{array}$ & $\begin{array}{l}-0,26^{*} \\
(-2,30)\end{array}$ \\
\hline $\begin{array}{l}\text { Alter (geteilt durch } \\
\text { 10) }\end{array}$ & $\begin{array}{l}-0,07 \\
(-1,01)\end{array}$ & $\begin{array}{l}-0,02 \\
(-0,61)\end{array}$ & $\begin{array}{l}-0,01 \\
(-0,54)\end{array}$ & $\begin{array}{l}1,99^{+} \\
(1,68)\end{array}$ & $\begin{array}{l}-0,14 \\
(-1,61)\end{array}$ & $\begin{array}{l}-0,14 \\
(-1,64)\end{array}$ \\
\hline Frau & $\begin{array}{l}-0,21 \\
(-0,99)\end{array}$ & $\begin{array}{l}-0,20 * \\
(-1,97)\end{array}$ & $\begin{array}{l}-0,15^{*} \\
(-2,21)\end{array}$ & $\begin{array}{l}0,12 \\
(0,03)\end{array}$ & $\begin{array}{l}-0,00 \\
(-0,02)\end{array}$ & $\begin{array}{l}-0,09 \\
(-0,35)\end{array}$ \\
\hline Eigentümer & $\begin{array}{l}-1,98^{* *} \\
(-8,15)\end{array}$ & $\begin{array}{l}-0,54 * * \\
(-4,72)\end{array}$ & $\begin{array}{l}-0,29 * * \\
(-3,86)\end{array}$ & $\begin{array}{c}18,25^{* *} \\
(4,43)\end{array}$ & $\begin{array}{l}-1,55^{* *} \\
(-5,19)\end{array}$ & $\begin{array}{l}-1,45 * * \\
(-4,83)\end{array}$ \\
\hline Konstante & $\begin{array}{l}18,23 * * \\
(24,51)\end{array}$ & $\begin{array}{l}17,66^{* *} \\
(50,21)\end{array}$ & $\begin{array}{l}13,52 * * \\
(58,20)\end{array}$ & $\begin{array}{l}263,83 * * \\
(20,92)\end{array}$ & $\begin{array}{l}50,59 * * \\
(55,03)\end{array}$ & $\begin{array}{l}39,51 * * \\
(42,67)\end{array}$ \\
\hline Korr. R-Quadrat & $\begin{array}{l}0,563 \\
2567\end{array}$ & $\begin{array}{l}0,679 \\
2566\end{array}$ & $\begin{array}{l}0,682 \\
2566\end{array}$ & $\begin{array}{l}0,444 \\
2563\end{array}$ & $\begin{array}{l}0,102 \\
2544\end{array}$ & $\begin{array}{l}0,066 \\
2544\end{array}$ \\
\hline
\end{tabular}

t-Werte in Klammern, ${ }^{+} \mathrm{p}<0,10,{ }^{*} \mathrm{p}<0,05,{ }^{*} \mathrm{p}<0,01$ 


\section{Literatur}

Ash, Michael, und T. Robert Fetter. 2004. Who lives on the wrong side of the environmental tracks? Evidence from the EPA's risk-screening environmental indicators model. Social Science Quarterly 85:441-462.

Atlas, Mark. 2002. Few and far between? An environmental equity analysis of the geographic distribution of hazardous waste generation. Social Science Quarterly 83:365-378.

BAFU, Bundesamt für Umwelt. 2009a. Lärmbelastung in der Schweiz. http://www.bafu.admin. $\mathrm{ch} /$ publikationen/publikation/01036/index.html?lang=de. Zugegriffen: 17. Juni 2009.

BAFU, Bundesamt für Umwelt. 2009b. Luftbelastung: Schadstoffkarten. http://www.bafu.admin. $\mathrm{ch} /$ luft/luftbelastung/schadstoffkarten/index.html?lang=de. Zugegriffen: 11. Feb. 2010.

BAFU, Bundesamt für Umwelt. 2009c. SonBase - Die GIS-Lärmdatenbank der Schweiz. http:// www.bafu.admin.ch/publikationen/publikation/01037/index.html?lang=de. Zugegriffen: 17 . Juni 2009.

BAFU, Bundesamt für Umwelt und Eidgenössische Materialprüfungs- und Forschungsanstalt EMPA. 2009. Nationales Beobachtungsnetz für Luftfremdstoffe (NABEL). http://www.empa. ch/plugin/template/empa/*/6174. Zugegriffen: 17. Juni 2009.

BAFU, Bundesamt für Umwelt und Cercl'Air, Schweizerische Gesellschaft der LufthygieneFachleute. 2009. Immissionswerte: Messdaten von nationalen, kantonalen und kommunalen Messstationen für Luftschadstoffe. http://www.bafu.admin.ch/luft/luftbelastung/blick_zurueck/01694/index.html?lang=de. Zugegriffen: 11. Feb. 2010.

Beck, Ulrich. 1986. Risikogesellschaft. Auf dem Weg in eine andere Moderne. Frankfurt a. M.: Suhrkamp.

Becker, Winfried, Bettina Kaiser, Sabine Luther, und Heike Otremba. 2008. Feuchtschäden in Wohnräumen: gesundheitliche, umweltbezogene und soziale Aspekte - Kleinräumige Erhebung aus dem Gesundheitsamt Bremen. Gesundheitswesen 70:636-639.

Bolte, Gabriele, und Martina Kohlhuber. 2008. Sozioökonomische Faktoren und Umweltbelastungen in Deutschland - aktueller Erkenntnisstand und exemplarische Analysen ausgewählter Umweltschadstoffe Teilprojekt A: Systematische Zusammenstellung der Datenlage in Deutschland. Publikationen des Umweltbundesamtes. Umweltbundesamt. Oberschleißheim, Bayerisches Landesamt für Gesundheit und Lebensmittelsicherheit. Ecological Economics 58:801-813.

Bolte, Gabriele, und Andreas Mielck, Hrsg. 2004. Umweltgerechtigkeit. Die soziale Verteilung von Umweltbelastungen. Gesundheitsforschung. Weinheim/München: Juventa Verlag.

Braun-Fahrländer, Charlotte. 2004. Die soziale Verteilung von Umweltbelastungen bei Kindern in der Schweiz. In Umweltgerechtigkeit. Die soziale Verteilung von Umweltbelastungen, Hrsg. Gabriele Bolte und Andreas Mielck, 155-173. Wenheim/München: Juventa.

Der Schweizerische Bundesrat. 1985. Luftreinhalte-Verordnung. http://www.admin.ch/ch/d/sr/ c814_318_142_1.html. Zugegriffen: 11. Feb. 2010.

Der Schweizerische Bundesrat. 1986. Lärmschutz-Verordnung. http://www.admin.ch/ch/d/sr/814 41/index.html. Zugegriffen: 16. Sep. 2009.

Die Schweizerische Post. 2003. Up to date: Postleitzahlenverzeichnis, Beschreibung der Angebote und Datenstruktur. Bern, Die Schweizerische Post, PostMail.

Diekmann, Andreas, und Reto Meyer. 2008. Schweizer Umweltsurvey 2007. Dokumentation und Codebuch. Zürich, Professur für Soziologie, ETH Zürich.

Ehrhardt-Martinez, Karen, Edward M. Crenshaw, und J. Craig Jenkins. 2002. Deforestation and the environmental Kuznets curve: A cross-national investigation of intervening mechanisms. Social Science Quarterly 83:226-243.

Elvers, Horst-Dietrich. 2007. Umweltgerechtigkeit als Forschungsparadigma der Soziologie. Soziologie. Forum der Gesellschaft für Soziologie 1:21-44. 
Evans, Gary.W., und Elyse Kantrowitz. 2002. Socioeconomic status and health: The potential role of environmental risk exposure. Annual Review of Public Health 23:303-331.

Grossman, Gene M., und Alan B. Krüger. 1995. Economic growth and the environment. The Quarterly Journal of Economics 110:353-377.

Grunenberg, Heiko, und Udo Kuckartz. 2003. Umweltbewusstsein im Wandel. Ergebnisse der UBAStudie. Umweltbewusstsein in Deutschland 2002. Opladen: Leske und Budrich.

Hornberg, Claudia, und Andrea Pauli. 2009. Umweltgerechtigkeit - die soziale Verteilung von gesundheitsrelevanten Umweltbelastungen. Dokumentation der Fachtagung vom 27.-28. Oktober 2008 in Berlin, Hrsg. Bundesministerium für Umwelt, Naturschutz und Reaktorschutz (BMU) und Umweltbundesamt (UBA) Bundesministerium für Umwelt. Eigenverlag, Universität Bielefeld.

Maschewsky, Werner. 2001. Umweltgerechtigkeit, Public Health und soziale Stadt. Frankfurt a. M.: VAS-Verlag für Akademische Schriften.

Maschewsky, Werner. 2004. Umweltgerechtigkeit - die Diskussion in den USA. In Umweltgerechtigkeit. Die soziale Verteilung von Umweltbelastungen, Hrsg. Gabriele Bolte und Andreas Mielck, 29-40. Wenheim: Juventa Verlag.

Mielck, Andreas, und Joachim Heinrich. 2002. Soziale Ungleichheit und die Verteilung umweltbezogener Exposition (Environmental Justice). Gesundheitswesen 64:405-416.

O'Neill, Marie, Michael Jerrett, Ichiro Kawachi, Jonathan I. Levy, Aaron J. Cohen, Nelson Gouveia, Paul Wilkinson, Tony Fletcher, Luis Cifuentes, und Joel Schwartz. 2003. Health, wealth, and air pollution: Advancing theory and methods. Environmental Health Perspectives 111:1861-1870.

Preisendörfer, Peter. 1999. Umwelteinstellungen und Umweltverhalten in Deutschland. Empirische Befunde und Analysen auf der Grundlage der Bevölkerungsumfragen: Umweltbewußtsein in Deutschland 1991-1998. Opladen: Leske + Budrich.

SAEFL, Swiss Agency for the Environment Forests and Landscape. 2003. Modelling of PM10 and PM2.5 ambient concentrations in Switzerland 2000 and 2010. Environmental Documentation No. 169, Air. Berne: Swiss Agency for the Environment Forests and Landscape.

SAEFL, Swiss Agency for the Environment Forests and Landscape. 2004. Modelling of NO2 and benzene ambient concentrations in Switzerland 2000 to 2020. Environmental Documentation No. 188, Air. Berne: Swiss Agency for the Environment Forests and Landscape.

Schuler, Martin, Pierre Dessemontet, und Dominique Joye. 2005. Die Raumgliederungen der Schweiz. Eidgenössische Volkszählung 2000. Neuenburg: Bundesamt für Statistik.

York, Richard, Eugene A. Rosa, und Thomas Dietz. 2003. Footprints on the earth: The environmental consequences of modernity. American Sociological Review 68:279-300.

Andreas Diekmann, 1951, Prof. Dr., Professor für Soziologie an der ETH Zürich. Forschungsgebiete: Theorie sozialer Kooperation, Umweltsoziologie, Bevölkerung, Methoden und Modelle, Experimentelle Spieltheorie. Veröffentlichungen: Trust and Reputation in Internet Auctions. In: Trust and Reputation (Hsrg. K. S. Cook et al.), New York 2009 (mit B. Jann und D. Wyder); Spieltheorie. Eine Einführung, Reinbek 2009 (2. Aufl. 2010). Benford's Law and Fraud Detection. Facts and Legends, erscheint in: German Economic Review, 2010 (mit B. Jann). Soziale Normen als Signale. Der Beitrag der Signaling-Theorie zur Erklärung sozialer Normen. Erscheint In: Soziologische Theorie kontrovers. 50. Sonderheft der Kölner Zeitschrift für Soziologie und Sozialpsychologie (Hrsg. G. Albert, S. Sigmund), 2010 (mit W. Przepiorka). 
Reto Meyer, 1974, Doktorand an der ETH Zürich. Forschungsgebiete: Umweltsoziologie und Methoden der Sozialwissenschaften, insbesondere Untersuchungen mit geokodierten Daten. Projekt: der Schweizer Umweltsurvey 2007. Veröffentlichungen: Umweltbewusstsein, Umwelthandeln und ökologische Risiken. In: Sozialbericht 2008 (Hrsg. C. Suter et al.) 2008 Zürich (m. A. Diekmann); Environmental Attitudes in Cross-National Perspective: A Multilevel Analysis of the ISSP 1993 and 2000. European Sociological Review 2010 (m. A. Franzen); Are the affluent prepared to pay for the planet? Explaining willingness to pay for public and quasi-private environmental goods in Switzerland. Population and Environment (m. U. Liebe, im Erscheinen). 